\title{
Multivariate Analysis of Gamma Spectra to Characterize Used Nuclear Fuel
}

\author{
Jamie Coble $^{\mathrm{a}, *}$, Christopher Orton ${ }^{\mathrm{b}}$, Jon Schwantes $^{\mathrm{b}}$ \\ ${ }^{a}$ University of Tennessee, 210 Pasqua Engineering Building, Knoxville, TN 37996, USA \\ ${ }^{b}$ Pacific Northwest National Laboratory, 902 Battelle Boulevard, Richland, WA 99352, USA
}

\begin{abstract}
The Multi-Isotope Process (MIP) Monitor provides an efficient means to monitor the process conditions in used nuclear fuel reprocessing facilities to support process verification and validation. The MIP Monitor applies multivariate analysis to gamma spectroscopy of key stages in the reprocessing stream in order to detect small changes in the gamma spectrum, which may indicate changes in process conditions. This research extends the MIP Monitor by characterizing a used fuel sample after initial dissolution according to the type of reactor of origin (pressurized or boiling water reactor; PWR and BWR, respectively), initial enrichment, burn up, and cooling time. Simulated gamma spectra were used to develop and test three fuel characterization algorithms. The classification and estimation models employed are based on the partial least squares regression (PLS) algorithm. A PLS discriminate analysis model was developed which perfectly classified reactor type for the three PWR and three BWR reactor designs studied. Locally weighted PLS models were fitted on-the-fly to estimate the remaining fuel characteristics. For the simulated gamma spectra considered, burn up was predicted with $0.1 \%$ root mean squared percent error (RMSPE) and both cooling time and initial enrichment with approximately $2 \%$ RMSPE. This approach to automated fuel characterization can be used to independently verify operator declarations of used fuel characteristics and to
\end{abstract}

\footnotetext{
* Corresponding author

Email address: jamie@utk.edu (Jamie Coble)
}

Preprint submitted to Nuclear Instruments and Methods in Physics Research AJanuary 6, 2017

(C) 2017. This manuscript version is made available under the Elsevier user license http://www.elsevier.com/open-access/userlicense/1.0/ 
inform the MIP Monitor anomaly detection routines at later stages of the fuel reprocessing stream to improve sensitivity to changes in operational parameters that may indicate issues with operational control or malicious activities.

Keywords: Used Nuclear Fuel, Fuel Characterization, Multivariate Analysis, Gamma Spectroscopy

\section{Introduction}

Current approaches to nuclear material control and accountancy (MC\&A) rely on destructive assay (DA) techniques, such as thermal ionization mass spectrometry, to deter and detect theft of special nuclear materials (SNM) at

5 nuclear reprocessing plants. Although these DA measurements are highly accurate and precise, they require extensive effort by skilled staff. While analytical results are being generated, significant quantities of SNM could be diverted from a large reprocessing facility. Additionally, the errors associated with material balances using these measurements scale with the size of accountancy tank. Without new methods to augment the current destructive assay-based MC\&A approaches, these errors may limit the size of a material balance area or of the overall reprocessing facility. Online, near-real-time process monitoring methods can be used in concert with traditional MC\&A measures to reduce the resources necessary to detect material diversion, improve the timeliness of detection and MC\&A decisions, and support large-scale used nuclear fuel reprocessing facilities.

The Multi-Isotope Process (MIP) Monitor is one approach to online process monitoring in nuclear fuel reprocessing facilities. The monitor uses multivariate analysis of gamma spectra collected at key points in reprocessing streams to detect small changes in the measured spectra that may indicate changes in process conditions. By targeting the full suite of gamma-emitting indicator isotopes, small, inexpensive, portable gamma detectors can be deployed throughout an existing facility to support the MIP Monitor. Because the MIP Monitor focuses on process monitoring, it could be used as an operator support tool for pro- 
cess control in reprocessing facilities or as a safeguards tool by the regulating authority; the same multivariate analysis techniques support either capability.

In past research, a database of training spectra covering normal operating conditions across a variety of used nuclear fuel types was used to train these multivariate methods $[1,2]$. This approach can identify gross changes in pro-

30 cess parameters, but small deviations may be obfuscated by the large number of conditions lumped into a single model. As the range of normal conditions included in training increases (e.g., by including fuel samples from many different reactors operating under different conditions), the sensitivity of the model to small deviations may decrease. This research extends the MIP Monitor by characterizing the used fuel samples at the initial dissolution stage. By characterizing the used fuel, multivariate models of normal behavior at later stages in the reprocessing stream can be trained specific to the conditions expected for the present used nuclear fuel sample. This will improve the sensitivity of the MIP Monitor to very small changes at later stages in the process. Additionally,

40 this automated fuel characterization can be used to verify operator declarations of used fuel inventories unobtrusively and independently, effectively in real time.

\subsection{Background}

The MIP Monitor is designed to track plant conditions indirectly and nondestructively by monitoring multiple gamma-emitting radioactive isotopes throughout an aqueous reprocessing system. The distribution of many fission and activation products is influenced by several operational variables, including acid concentration, TBP concentration, temperature, and used fuel characteristics (e.g. reactor type, burn up, cooling time). For a consistent industrial process with reproducible conditions, the distribution of each isotope between the organic and aqueous phases should be relatively constant. Consequently, the distribution of isotopes in both product and waste streams at each stage in the facility should be relatively reproducible (within normal industrial variations), resulting in a signature of "normal" process conditions. Under abnormal conditions (such as those expected under some protracted diversion scenarios), the 
distribution of isotopes in the various streams may change measurably $[3,4]$. The MIP Monitor approach capitalizes on these expected changes in the distribution of gamma-emitting isotopes in the aqueous and organic phases to serve as an indication of "off-normal" conditions in the process chemistry. The MIP Monitor relies on gamma detectors that can be deployed at key locations ${ }_{60}$ throughout an existing facility. Multivariate analysis of gamma spectra is used to automatically and autonomously process the spectra from these detectors in real time, continuously monitoring for indications of changing stream conditions. Initial simulations and bench-scale experiments reported in prior work have documented the performance and potential of the MIP Monitor $[1,2]$. This

65 approach can identify gross changes in process parameters, but small deviations may be obfuscated by the range of normal conditions, resulting in part from the variety of used nuclear fuel that can be reprocessed in a given facility. As the range of normal conditions included in training increases, the sensitivity of the model to small deviations and anomalies may decrease. This research extends the MIP Monitor by characterizing the used fuel samples after initial dissolution according to the reactor type (pressurized or boiling water reactor; PWR or BWR, respectively), initial enrichment, burn up, and cooling time. This characterization allows for independent verification of operator-declared fuel inventories. Additionally, multivariate models used to monitor later stages

75 in the reprocessing facility can be trained using only gamma spectra of fuel samples similar to the current one according to the estimated fuel characteristics. This will improve the sensitivity of the MIP Monitor to very small changes that may be indicative of undesirable process changes or diversion of special nuclear material.

\subsection{Organization of the Article}

This article presents a framework for multivariate analysis of gamma spectra for the purpose of characterizing used nuclear fuel. The following section describes the multivariate analysis methodology used: partial least squares regression, as well as two special cases, partial least squares discriminate anal- 
ysis and locally weighted partial least squares regression. The specific locally weighted partial least squares implementation described here differs from other approaches in the information used to identify local observations and calculate weights. This is followed by a summary of the simulation of used nuclear fuel composition and resulting gamma spectra in Section 3. Results of the simulation, modeling, and characterization are given in Section 4. Finally, conclusions are drawn and discussion of the expected efficacy of the method on real-world data is given.

\section{Methodology}

Several multivariate analysis methods were investigated for application in in the following sections, preceded by a brief discussion of the preprocessing methods used to prepare gamma spectra for modeling. 


\subsection{Multivariate Analysis Algorithms}

115

plify calculations by removing systematic deviations, such as noise, mean signal values, or signal background, that do not provide useful information about the process or system. Data preprocessing can be performed within the variables or within the observations. Observations can be normalized to reduce the effect this would be necessary if counting time or detector geometry is allowed to vary between observations; however, in this study the counting time and geometry are kept constant, and so observation intensity is retained because it is expected to contain important information for the used fuel characterization. In the analof each variable (energy channel) in the training data is subtracted from each observation of that variable (energy channel) to give a post-processed variable mean of zero in the training data. This mean centering removes the large, but uninteresting, offset of the channel average value from zero while maintaining This is particularly important for PCA and PLS analysis, because these methods identify underlying components that capture data variation from the origin. If the mean values of each variable are not removed from the training data, the fitted components will attempt to capture that variation; typically the first the training data. Other data preprocessing methods can be applied to reduce noise and maximize the signal-to-noise ratio (SNR). The simulated spectra used in this work are noise-free, so no noise analysis is investigated here. In a deployed system, noise analysis and reduction, as well as background subtraction,

\subsubsection{Partial Least Squares Regression}

Partial least squares regression (PLS) was originally developed in the field of econometrics, but has found more ubiquitous application in chemometrics. 
Practically, its application is not limited to chemical analyses; PLS is a superis the training output data. Because the rank of $y$ remains unchanged through PLS model development, the maximum number of LVs that can be identified is equal to the rank of $X$. Typically, however, fewer LVs are retained in the model 


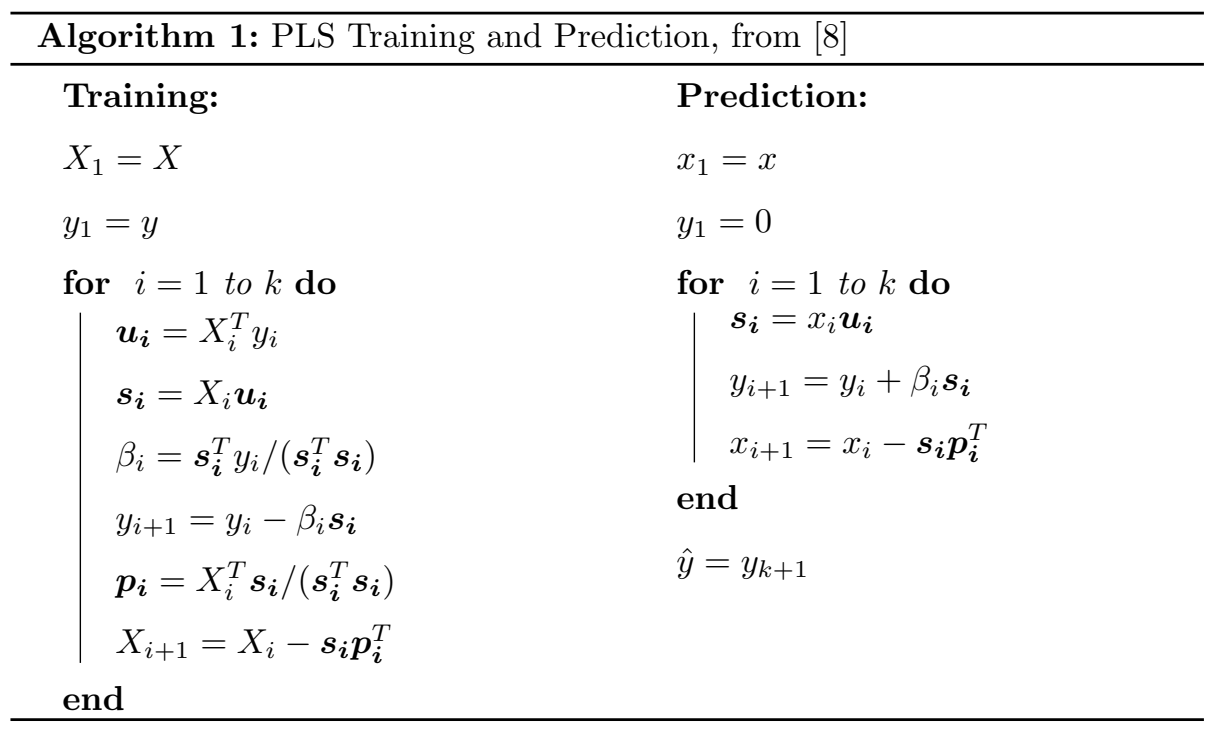

because, as in PCA, the later LVs likely contain primarily noise and information unuseful for predicting $y$. The algorithm shown selects some preselected number of LVs, $k$, based on prior knowledge of the true dimensionality of the system. Another approach is to select LVs until the output residual (the unexplained information in $y$, given by $y_{i+1}$ ) is smaller than some specified threshold.

In addition to the traditional PLS algorithm, two variations are used in this research: PLS discriminate analysis for classification and locally weighted PLS for estimation. These variations are described in the following subsections.

\subsubsection{PLS Discriminate Analysis}

The PLS algorithm given in Algorithm 1 can be easily adapted for classification purposes, commonly known as PLS discriminate analysis (PLSDA) [9]. In PLSDA, the output is a vector of binary dummy variables indicating whether the observation belongs to a specific class according to equation 1 .

$$
y_{i j}= \begin{cases}1 & \text { if obs } i \in \operatorname{class} j \\ 0 & \text { otherwise. }\end{cases}
$$

With this encoding, the PLS algorithm will preferentially select between- 
group variance that discriminates between classes. In the prediction step, the membership of an observation in each class is estimated; these continuous measures of membership are converted to binary numbers by either applying the threshold or a winner-take-all approach.

\subsubsection{Locally Weighted PLS}

Conventional PLS is an inherently linear modeling technique, which can limit considered approximately linear in some small neighborhood about a point. Locally weighted models are often used to apply linear methods to nonlinear data by building local models around each new prediction point. Locally weighted PLS (LWPLS) can preserve the globally nonlinear information by creating locally linear PLS models as new observations are made [10, 11, 12].

LWPLS differs from conventional PLS by the introduction of a weighting matrix, $W$, that determines the relative importance of each training observation in determining LVs and regression parameters. In the LWPLS training algorithm, $\boldsymbol{u}_{\boldsymbol{i}}, \beta_{i}$, and $\boldsymbol{p}_{\boldsymbol{i}}$ are calculated according to the formulae in equation 2052.

$$
\begin{aligned}
& \boldsymbol{u}_{\boldsymbol{i}}=X^{T} W y_{i} \\
& \beta_{i}=\boldsymbol{s}_{\boldsymbol{i}}^{T} W y_{i} /\left(\boldsymbol{s}_{\boldsymbol{i}}^{T} W \boldsymbol{s}_{\boldsymbol{i}}\right), \text { and } \\
& \boldsymbol{p}_{\boldsymbol{i}}=X_{i}^{T} W \boldsymbol{s}_{\boldsymbol{i}} /\left(\boldsymbol{s}_{\boldsymbol{i}}^{T} W \boldsymbol{s}_{\boldsymbol{i}}\right),
\end{aligned}
$$

where $W$ is a diagonal matrix that contains the weight for each of the exemplar input/output pairs in $X$ and $y$. The weights can be defined in any way that indicates the similarity of a new observation to the historic (exemplar) data. A Gaussian kernel is commonly used to convert the distance between the new query input and an exemplar input to a similarity or weight $[10,11]$. In this approach, the weight for an exemplar vector with respect to a new query vector 
is given by equation 3 :

$$
w_{i}=e^{-d_{i}^{2} / h^{2}},
$$

where $h$ is the kernel bandwidth and the distance, $d$, is typically given by the Euclidean distance (equation 4):

$$
d_{i}=\sqrt{\sum_{k=1}^{N}\left(x_{i k}-q_{k}\right)^{2}},
$$
for the query observation, and $c_{i, k}$ is the known value of the $k^{\text {th }}$ characteristic for the $i^{\text {th }}$ exemplar. The similarity for binary characteristics (i.e., reactor type, 
$R x)$ is calculated as in equation 6 .

$$
w\left(\hat{c}_{R x}, c_{i, R x}\right)= \begin{cases}1 & \hat{c}_{R x}=c_{i, R x} \\ 0 & \hat{c}_{R x} \neq c_{i, R x}\end{cases}
$$

235 match the exemplar reactor type. The similarity for continuous characteristics (initial enrichment, burn up, cooling time) is calculated using the Gaussian kernel in equation 3. The kernel bandwidth, $h$, is optimized for each characteristic individually, $h_{k}$, based on the range of values of the characteristic and the additional predictive power gained by including that information in the model. As $h_{k}$ approaches zero, equation 3 approaches the binary weighting in equation 5 , effectively producing a nearest-neighbor matching; conversely, as $h_{k}$ becomes large relative to the expected distance between query and exemplar features, the weights all asymptotically approach one, and the LWPLS algorithm converges to the traditional PLS model.

\subsection{Fuel Characterization Algorithms}

Three characterization algorithms were compared. The first algorithm performed all characterizations in parallel, meaning that fuel type, burn up, enrichment, and cooling time were characterized using only the measured gamma spectrum (Figure 1). The second algorithm, referred to as Algorithm $\mathrm{A}$ and shown in Figure 2, first classified the fuel type (PWR or BWR), and then used reactor type-specific PLS models to predict burn up, enrichment, and cooling time. Finally, the third algorithm (Algorithm B, Figure 3) is fully serial, where the fuel type is first classified; then the burn up is estimated using a reactor to train a LWPLS to predict cooling time; and initial enrichment is finally estimated using an LWPLS model weighted according to the estimated reactor type, burn up, and cooling time. Because the weights in each subsequent model depend on the fuel characteristics previously estimated, the order of evaluation 


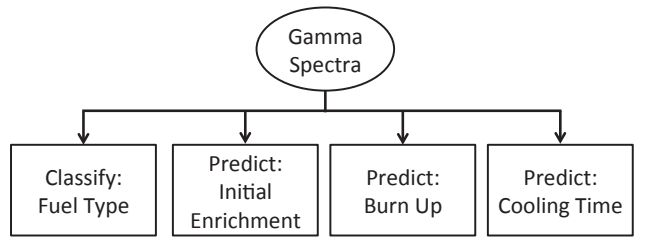

Figure 1: Flow Diagram for Parallel Analysis

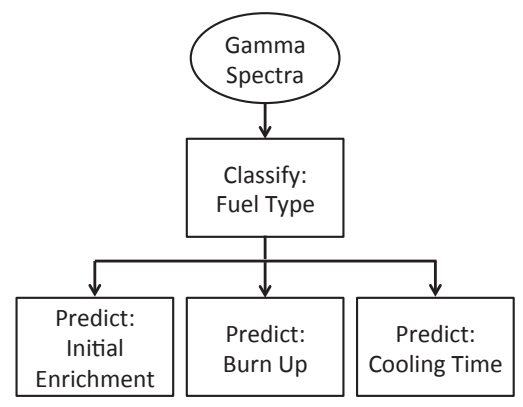

Figure 2: Flow Diagram for Algorithm A

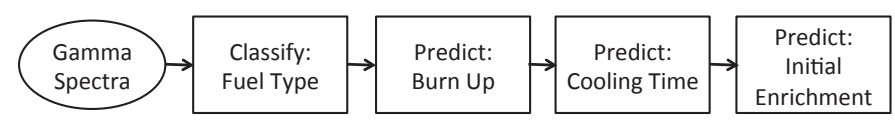

Figure 3: Flow Diagram for Algorithm B

algorithm was selected through trial-and-error by testing each permutation of evaluation order on a set of test data to optimize the overall performance across all four fuel characteristics, represented by the average root mean squared percent error across the four characteristics.

\section{Application to Simulated Spectra}

The data used to develop and test the analysis methodology were generated using ORIGEN-ARP version 5.1.01 and ENDF/B-VI nuclear data [13]. The development data consisted of three PWR and three BWR reactor designs, six initial enrichment values evenly distributed among typical commercial values, four cooling times, and nine discharge burn up values (Table 1). Each simulation used three cycles of equal length (18 months) with $100 \%$ availablility. The 
first two cycles are followed by a 30-day outage, and the third cycle is followed the relevant fuel cooling time. The cooling times were chosen based on the storage capacity at Rokkasho Reprocessing Plant, a commercial fuel reprocessing facility in Japan [14]. A consistent reactor operating history was maintained in this study to evaluate the ability to estimate fuel characteristics alone; the effects of operating history on the efficacy and accuracy of this approach should be investigated in future work. The 200 nuclides with the highest activity in the ORIGEN-ARP output of each fuel sample were retained and used to simulate the gamma spectra of the fuels [15]. This resulted in 218 unique nuclides, although any single simulation had only 200 non-zero nuclide concentrations. Benchmark studies of ORIGEN-ARP/ORIGEN-S [16, 17, 18] were reviewed to gauge the accuracy of the nuclide simulations, which would in turn indicate the accuracy of the simulated gamma spectra. The results of this benchmarking evaluation are available in [19]. Only benchmarking studies evaluating used fuel similar to those simulated here were considered. Of the fourteen nuclides evaluated for accuracy, most were accurate to $10 \%$, although ${ }^{126} \mathrm{Sn}$ and ${ }^{79} \mathrm{Se}$ have reported error of 202.0 and $980.0 \%$, respectively. These large errors are typically attributed to inaccurate cross section data used for simulation. Despite the large errors in these and potentially other key nuclides, the overall trends in between-sample spectra variations are thought to be representative of the variations expected in practice. Artifacts in the simulations caused by the miscalculation of key nuclides limit our ability to directly apply the developed models to measured UNF spectra. Because the MIP Monitor analysis capitalizes on these between-sample variations for fuel characterization instead of the intensity of any specific peak or set of peaks, the proof-of-principle demonstration with these data suggest that this approach is viable for real-world deployment if appropriate measured spectra are available to train the classification and estimation models.

The 200 most abundant nuclides for each of the simulated fuel samples are input to SYNTH, a program developed at Pacific Northwest National Laboratory (PNNL) to simulate the measured gamma spectrum for a given source and 
Table 1: Used Fuel Characteristics for Data Simulation. Fuel and gamma spectra are simulated for each combination of reactor design, BU, IE, and CT.

\begin{tabular}{c|lc|c|c}
\hline $\begin{array}{c}\text { Reactor } \\
\text { Design }\end{array}$ & \multicolumn{2}{|c|}{$\begin{array}{c}\text { Burn Up } \\
\text { (MWd/kgU) }\end{array}$} & $\begin{array}{c}\text { Initial } \\
\text { Enrichment (\%) }\end{array}$ & $\begin{array}{c}\text { Cooling Time } \\
\text { (years) }\end{array}$ \\
\hline CE14x14 (PWR) & 20 & 36 & 2.5 & 2 \\
S14x14 (PWR) & 25 & 40 & 3.0 & 3 \\
W17x17(PWR) & 30 & 45 & 3.5 & 4 \\
Atrium9x9 (BWR) & 34 & 50 & 4.0 & 5 \\
GE7x7 (BWR) & 35 & & 4.5 & \\
SVEA64 (BWR) & & & 5.0 & \\
\hline
\end{tabular}

Table 2: HPGe Detector and Electronics Characteristics in SYNTH Simulation of Gamma Spectra

\begin{tabular}{cll}
\hline & Thickness & $0.5 \mathrm{~mm}$ \\
End Cap & Material & Aluminum \\
& Spacing & $0.67 \mathrm{~mm}$ \\
\hline Dead Layer & Thickness & $0.67 \mathrm{~mm}$ \\
\hline \multirow{2}{*}{ Sensor } & Diameter & $6.67 \mathrm{~cm}$ \\
& Length & $6.67 \mathrm{~cm}$ \\
& Efficiency & $66.60 \%$ \\
& Resolution @ $1332 \mathrm{keV}$ & $1.67 \mathrm{keV}$ \\
\hline Counting Parameters & Gero & $0 \mathrm{keV}$ \\
& Quad & $1 \mathrm{keV} / \mathrm{ch}^{2}$ \\
\hline Full Scale Energy & Channels & $0 \mathrm{keV} / \mathrm{ch}^{2}$ \\
\hline
\end{tabular}

detector configuration [20]. For this analysis, the gamma source was treated as a point source $25 \mathrm{~cm}$ from a coaxial high-purity germanium (HPGe) detector; pertinent characteristics of the detector and electronics are listed in Table 2. 
The 1296 simulated gamma spectra were divided into three independent sets for model development and testing. The training data set, which is used to train the various PLS-based characterization models, includes 722 spectra that cover the range of simulated values in each channel and the range of fuel characteristics. This coverage is important to limit the extrapolation that the model must perform when presented with new spectra; empirical models generally should not be relied on to extrapolate outside of their training region. The test data contains 324 spectra, which are used to optimize the trained PLS-based models for the number of latent variables and the bandwidth used to determine weights in LWPLS models. The remaining 324 spectra are used to characterize the performance of the fuel characterization algorithm, the so-called validation data. The characterization results presented in the next section are evaluated on the validation data.

\section{Results}

Three primary algorithms were evaluated for characterization of used nuclear fuel according to reactor type, initial enrichment, burn up, and cooling time, as described in section 2.2. The results of each of these algorithms are evaluated on accuracy and runtime. The MIP Monitor must be able to make accurate characterization in near-real-time, in order to improve MC\&A and process monitoring at reprocessing facilities. Essentially, the MIP Monitor must evaluate key fuel characteristics correctly and before the next spectrum is available.

The PLS algorithms used here are data compression techniques that combine related and redundant information across multiple spectrum channels into underlying LVs. The simulated HPGe gamma spectra include 4096 energy channels, effectively 4096 input variables for the multivariate analysis. These channels are compressed into a smaller subset of LVs, each of which contains some portion of each energy channel. The PLSDA model developed for reactor type classification uses thirty-six latent variables. The PLS and LWPLS models developed for estimating the IE, BU, and CT include from sixteen to fifty LVs. 
All of these models provide a significant reduction of dimensionality from the original 4096 energy channels. The accuracy results presented below suggest that the true dimensionality of these data is significantly lower than the number of energy channels available; these extra dimensions can confound other multivariate regression techniques that lack the data compression of PLS.

\subsection{Characterization Accuracy}

Three algorithms are evaluated for characterizing used nuclear fuel: parallel analysis (Figure 1), Algorithm A (Figure 2), and Algorithm B (Figure 3). In the parallel analysis, each of the four primary characteristics is evaluated in parallel using only the measured gamma spectrum. In Algorithm A, the reactor type is first classified, and the remaining three characteristics are estimated using reactor type-specific PLS models. In the final approach, Algorithm B, the classification is fully serial, where the classification results from the previous stage are used to develop a new LWPLS model on the fly to evaluate the fuel characteristic at the current stage. The accuracy of each of these algorithms is compared according to the root mean squared percent error (RMSPE), which is calculated according to equation 7 :

$$
R M S P E=\frac{\sqrt{E\left[(\hat{y}-y)^{2}\right]}}{E[y]} \times 100 \%
$$

where $\hat{y}$ is the prediction of a specific fuel characteristic whose true value is $y$ and $E[\cdot]$ is the expected value.

The accuracy of each of the three fuel characterization algorithms for predicting the four key fuel characteristics is summarized in Table 3. Here, the results are presented for each algorithm predicting the characteristic for all of the validation data and for the PWR and BWR fuels separately, as well as the overall results combining both PWR and BWR fuels. For all evaluated characterization algorithms, a PLSDA model was trained that perfectly classifies used fuel according to reactor type (PWR or BWR); the same model is used in each algorithm. Figure 4 shows the scores of the test data on latent variables 1 and 2 of the PLSDA classification model. As the figure shows, there is significant 
overlap between the two reactor types in two dimensions; the discrimination is not visually obvious in two or three dimensions. As discussed previously, thirtysix latent variables are necessary to accurately discriminate the reactor type. Algorithm A shows a significant improvement over the parallel analysis for the data evaluated, with improvements in accuracy of a factor of two to four for prediction of initial enrichment, burn up, and cooling time. Algorithm B shows further improvement over Algorithm A for prediction of initial enrichment and cooling time. Due to the weighting scheme used, the LWPLS model used to predict the burn up in Algorithm B is effectively identical to the reactor typespecific model used in Algorithm A. The LWPLS models used in later stages of Algorithm B (i.e., prediction of initial enrichment and cooling time) make use of the additional fuel characteristics predicted in earlier stages of the algorithm.

The accuracy results achieved in this evaluation are unrealistically low, because the simulated data do not contain measurement noise or random, normal perturbations in the fuel isotopics. However, these results suggest that fuel characterization of dissolved used nuclear fuel samples through multivariate analysis of gamma spectra can be effective.

\subsection{Runtime Results}

The MIP Monitor is intended to work in real-time (or near-real-time) to monitor reprocessing streams. Generally, the monitor is expected to use 30second spectra (or longer); therefore, the multivariate analysis methods employed should complete predictions in under 30 seconds to provide information in effectively real-time. This implementation was evaluated on a $3.40 \mathrm{GHz}$ Intel Pentium 4 processor. Execution speed for a single sample was evaluated by averaging the elapsed time for 324 validation samples. The MATLAB implementation averaged 1.85 seconds per evaluation; the model evaluation can be performed faster than the expected sampling time, providing real time fuel characterization.

\section{Conclusion}


Table 3: Fuel Characterization Error for Parallel Analysis, Algorithm A, and Algorithm B. Error is given as RMSPE (\%, top) and 95-percentile ranges of error in engineering units (bottom).

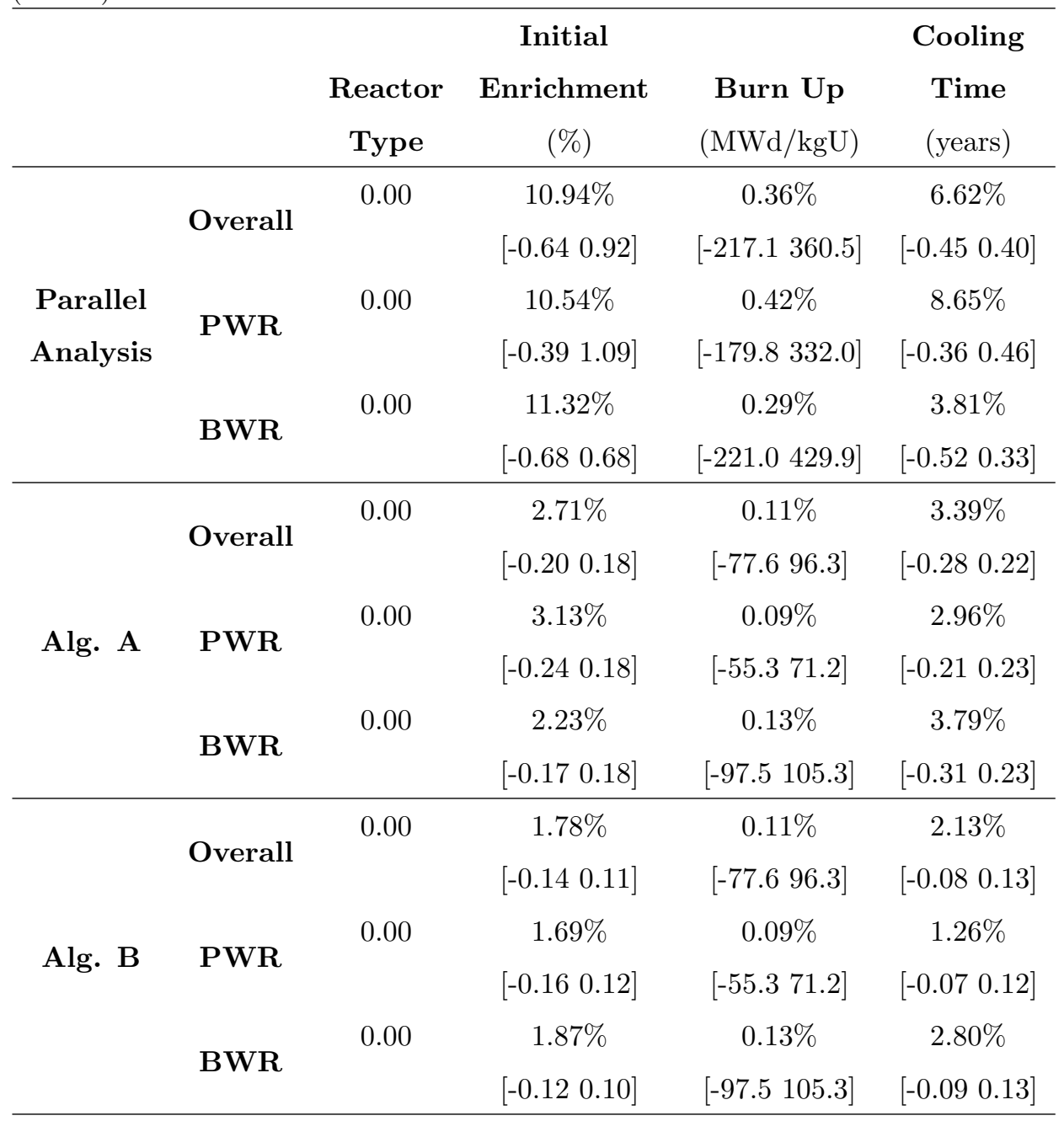

This investigation extends the previously developed MIP Monitor approach for process monitoring and MC\&A at used nuclear fuel reprocessing facilities to support fuel characterization at early stages in the reprocessing stream through multivariate analysis of gamma spectra. This approach to used fuel characterization provides an independent verification of operator declarations and can improve the ability of the MIP Monitor to detect anomalous conditions at later 


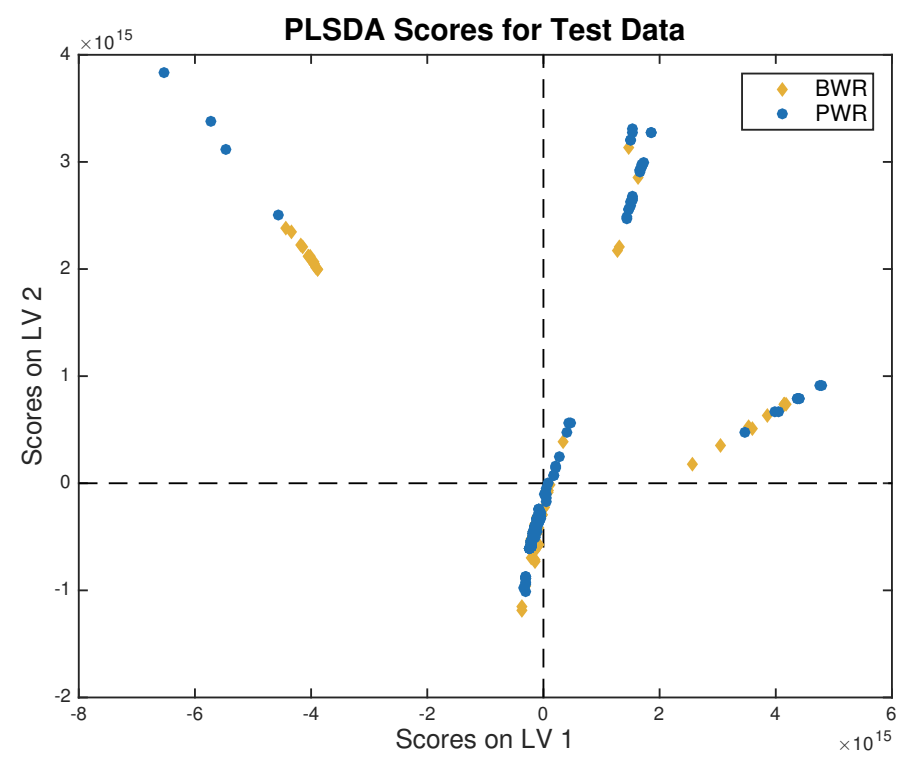

Figure 4: PLSDA Scores on Latent Variable 1 and 2 for the Test Data

stages in the fuel reprocessing stream. This initial proof-of-concept study used simulated data from ORIGEN-ARP and SYNTH to develop models and algo-

rithms for fuel characterization. The performance of the developed characterization algorithms, both in terms of accuracy and computational speed, suggests that similar analyses could be successfully developed for in situ fuel characterization at an operating aqueous reprocessing facility. The proposed framework for fuel characterization is expected to be generally applicable, but new models and characterization algorithms will need to be developed using experimental or operational spectra.

This initial proof-of-principle study investigates a "best case" scenario for the proposed fuel characterization and declaration verification framework. Spectra are simulated for high-resolution gamma detectors (HPGe), which are unlikely to be deployed in operating fuel reprocessing facilities. Shielding, containers, and conduits that may attenuate gamma radiation are not included in the spectra simulation. Further, background contributions to the spectra are neglected. Initial investigations at the H-Canyon facility at Savannah River Site suggest 
that background correction and spectrum alighment will be necessary preprocessing steps for measurements made at multiple locations within the facility [21]. These same data collection campaigns have successfully deployed $\mathrm{NaI}(\mathrm{Ti})$ detectors at various stages throughout the facility, suggesting that cyrogenicallycooled, high-resolution detectors will not be necessary in a deployment scenario. A targeted campaign to collect both gamma spectra and meta-data on a variety of typical used nuclear fuel samples at initial dissolution will be necessary to develop and fully test the proposed characterization framework.

\section{Acknowledgment}

The authors would like to thank Kenneth Dayman at the University of Texas at Austin for supplying the simulated used nuclear fuel nuclide activities.

This work described here was funded by the US Department of Energy Office of Nuclear Energy (DOE-NE) through the MPACT campaign of the Fuel Cycle Technologies research program. The work was performed at Pacific Northwest National Laboratory, operated by Battelle for the US Department of Energy.

\section{References}

[1] C. R. Orton, C. G. Fraga, R. N. Christensen, J. M. Schwantes, Proof of concept simulations of the multi-isotope process monitor: An online, nondestructive, near-real-time safeguards monitor for nuclear fuel reprocessing facilities, Nuclear Instruments and Methods in Physics Research Section A: Accelerators, Spectrometers, Detectors and Associated Equipment 629 (1) (2011) 209-219. doi:10.1016/j.nima.2010.10.024.

URL http://www.sciencedirect.com/science/article/pii/ S0168900210022588

[2] C. R. Orton, C. G. Fraga, R. N. Christensen, J. M. Schwantes, Proof of concept experiments of the multi-isotope process monitor: An online, nondestructive, near real-time monitor for spent nuclear fuel reprocessing 
facilities, Nuclear Instruments and Methods in Physics Research Section A: Accelerators, Spectrometers, Detectors and Associated Equipment 672 (2012) 38-45. doi:10.1016/j.nima.2011.12.083.

URL http://www.sciencedirect.com/science/article/pii/

[3] L. Smith, J. Schwantes, M. Douglas, J. Ressler, C. Durst, C. Orton, R. Christensen, Next-generation online mc\&a technologies for reprocessing plants, in: Global 2007: Advanced Fuel Cycles and Systems, Boise, ID, 2007.

[4] C. R. Orton, J. M. Schwantes, C. G. Fraga, M. Douglas, R. Christensen, Experimental validation of the multi-isotope process monitor concept, in: Conference Proceedings of GLOBAL 2009 "The Nuclear Fuel Cycle: Sustainable Options \& Industrial Perspectives", Paris, France, 2009.

[5] P. Geladi, B. R. Kowalski, Partial least-squares regression: A tutorial, Analytica Chimica Acta 185 (0) (1986) 1-17. doi: 10 . 1016/0003-2670 (86) 80028-9.

URL http://www.sciencedirect.com/science/article/pii/ 0003267086800289

[6] H. Hotelling, Analysis of a complex of statistical variables into principal components, Journal of Educational Psychology 24 (6) (1933) 417-441. doi:10.1037/h0071325.

[7] H. Hotelling, Analysis of a complex of statistical variables into principal components, Journal of Educational Psychology 24 (7) (1933) 498-520. doi: $10.1037 / \mathrm{h} 0070888$.

[8] H. Hoffmann, S. Schaal, S. Vijayakumar, Local dimensionality reduction for non-parametric regression, Neural Processing Letters 29 (2) (2009) 109131. doi:10.1007/s11063-009-9098-0.

URL http://dx.doi.org/10.1007/s11063-009-9098-0 
[9] M. Barker, W. Rayens, Partial least squares for discrimination, Journal of Chemometrics 17 (3) (2003) 166-173. doi:10.1002/cem.785.

URL http://dx.doi.org/10.1002/cem.785

[10] S. Schaal, C. G. Atkeson, S. Vijayakumar, Real-time robot learning with locally weighted statistical learning (April 24-28, 2000 2000).

[11] S. Kim, M. Kano, H. Nakagawa, S. Hasebe, Estimation of active pharmaceutical ingredients content using locally weighted partial least squares and statistical wavelength selection, International Journal of Pharmaceutics 421 (2) (2011) 269-274. doi:10.1016/j.ijpharm.2011.10.007.

URL http://www.sciencedirect.com/science/article/pii/ S0378517311009021

[12] J. Liu, D.-S. Chen, M.-W. Lee, Adaptive soft sensors using local partial least squares with moving window approach, Asia-Pacific Journal of Chemical Engineering 7 (2012) S134-S144. doi:10.1002/apj. 663.

URL http://dx.doi.org/10.1002/apj.663

[13] S. M. Bowman, L. C. Leal, Origen-arp: Automatic rapid process for spent fuel depletion, decay, and source term analysis, Report, U.S. Nuclear Regulatory Commission (2000).

[14] P. Durst, R. Wallace, I. Therios, M. Ehinger, R. Bean, D. Kovacic, A. Dougan, K. Tolk, B. Boyer, Advanced safeguards approaches for new reprocessing facilities.

[15] K. J. Dayman, J. B. Coble, C. R. Orton, J. M. Schwantes, Characterization of used nuclear fuel with multivariate analysis for process monitoring, Nuclear Instruments and Methods in Physics Research Section A: Accelerators, Spectrometers, Detectors and Associated Equipment 735 (2014) 624 - 632. doi:http://dx.doi.org/10.1016/j.nima.2013.09. 056. 
[16] I. Gauld, K. Litwin, Verification and validation of the origen-s code and nuclear data libraries, Report, Atomic Energy of Canada Limited (1995). URL http://www.ornl.gov/sci/scale/pubs/rc1429.pdf

[17] L. Leal, O. Hermann, S. Bowman, C. Parks, Automatic rapid process for the generation of problem-dependent sas $2 \mathrm{~h} /$ origen-s cross-section libraries, Nuclear Technology 127 (1) (1999) 1-23.

[18] I. C. Gauld, B. D. Murphy, Updates to the origen-s data libraries using endf/b-vi, fendl-2.0, and eaf-99 data, Report, Oak Ridge National Laboratory (2004).

[19] J. B. Coble, C. R. Orton, J. M. Schwantes, Automated characterization of spent fuel through the multi-isotope process (mip) monitor, Report, Pacific Northwest National Laboratory (2012).

[20] W. Hensley, A. McKinnon, H. Miley, M. Panisko, R. Savard, Synth: a spectrum synthesizer, Journal of Radioanalytical and Nuclear Chemistry 193 (2) (1995) 229-237.

[21] N. Shoman, J. B. Coble, D. M. Meier, Experimental performance of the multi isotope process monitor, in: Transactions of the 2015 ANS Winter Meeting and Technology Expo, 2015. 